The following paper was presented at The 9th Workshop on Disfluency in Spontaneous Speech (DiSS 2019) held at ELTE Eötvös Loránd University in Budapest, Hungary on 12-13 September, 2019.

Title: $\quad$ Segment prolongation in Hebrew

Author(s): $\quad$ Vered Silber-Varod, Mária Gósy and Robert Eklund

Abstract: In this paper we study segment prolongations (PRs), a type of disfluency sometimes included under the term "hesitation disfluencies", in Hebrew. PRs have previously been studied in a number of other languages within a comprehensive speech disfluency framework, which is applied to Hebrew in the current study. For the purpose of this study we defined Hebrew clitics, such as conjunctions, articles, prepositions and so on, as words. The most striking difference between Hebrew and the previously studies languages is how restricted PRs seem to be in Hebrew, occurring almost exclusively on word-final vowels. The most frequently prolonged vowel is [e]. The segment type does not affect PRs' duration. We found significant differences between men and women regarding the frequency of PRs.

DOI: https://doi.org/10.21862/diss-09-013-silb-etal

Citation (JIPA): Silber-Varod, Vered, Mária Gósy \& Robert Eklund. 2019. Segment prolongation in Hebrew. In: R. L. Rose \& R. Eklund (eds.), Proceedings of DiSS 2019, The 9th Workshop on Disfluency in Spontaneous Speech, 12-13 September, 2019, Budapest, Hungary, 47-50.

The complete proceedings for DiSS 2019 are available as follows.

ISBN:

978-963-489-063-8

DOI: https://doi.org/10.21862/diss-09

DiSS 2019 was sponsored by The Faculty of Humanities, ELTE Eötvös Loránd University and the International Speech Communication Association (ISCA).
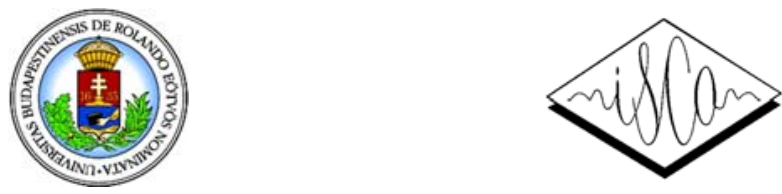


\title{
Segment prolongation in Hebrew
}

\author{
Vered Silber-Varod ${ }^{1}$, Mária Gósy ${ }^{2}$ and Robert Eklund ${ }^{3}$ \\ ${ }^{1}$ Open Media and Information Lab (OMILab), The Open University of Israel, Israel \\ ${ }^{2}$ Dept. of Phonetics, Research Institute for Linguistics, Hungarian Academy of Sciences, Budapest, Hungary \\ ${ }^{3}$ Dept. of Culture and Communication, Linköping University, Sweden
}

\begin{abstract}
In this paper we study segment prolongations (PRs), a type of disfluency sometimes included under the term "hesitation disfluencies", in Hebrew. PRs have previously been studied in a number of other languages within a comprehensive speech disfluency framework, which is applied to Hebrew in the current study. For the purpose of this study we defined Hebrew clitics, such as conjunctions, articles, prepositions and so on, as words. The most striking difference between Hebrew and the previously studies languages is how restricted PRs seem to be in Hebrew, occurring almost exclusively on wordfinal vowels. The most frequently prolonged vowel is [e]. The segment type does not affect PRs' duration. We found significant differences between men and women regarding the frequency of $P R s$.
\end{abstract}

\section{Introduction}

Prolongation (PR), long being studied in stuttered speech as a characteristic phenomenon, was found to be very common in non-pathological speech, as a disfluency phenomenon that is used when a speaker is hesitating (Eklund \& Shriberg, 1998; Eklund, 2001, 2004: 163), i.e. when a syllable or a speech segment in a word is produced unusually long. Although this is similar to (what is perhaps most commonly called) filled pauses (FPs) in that both are durational, PRs have been shown to differ from FPs in some respects (e.g. Eklund, 2001). Previous studies of PRs have covered a wide variety of languages that are taxonomically similar, such as English and Swedish (Eklund \& Shriberg, 1998), or taxonomically different, such Swedish and Tok Pisin (Eklund, 2001, 2004), Japanese (Den, 2003), and Mandarin Chinese (Lee et al, 2004). Other languages studied include German (Betz, Eklund \& Wagner, 2017), and Hungarian (Gósy \& Eklund, 2018).

One characteristic of PRs that have been discussed in those studies is how they are distributed in the word in which they appear. Simply put, a segment subjected to prolongation (above normal duration, relative to speech rate), can appear in three basic positions:

- Word initial, e.g. "ssssegment" [s:egment]

- Word medial, e.g. "segmmmmment" [segm:ent]

- Word final, e.g. "segmen t" [segment:]
Eklund and Shriberg (1998) reported for American English and Swedish, a 30-20-50\% distribution, for initial-medial-final positions, respectively. What made these figures interesting, however, were studies of other languages. Eklund (2001; 2004: 251) reported $15-0-85 \%$ in Tok Pisin; Den (2003) reported 0-5-95\% for Japanese; and Lee et al. (2004) reported 4-1-95\% for Mandarin Chinese.

The interesting difference was that Japanese, Mandarin and Tok Pisin exhibit both much less complex syllable structures, and even less complex phonotactics, as compared to English and Swedish. This led Eklund (2004: 251) to coin the 'morphology matters hypothesis', although Eklund later rephrased this (somewhat misleading term) as 'phonotactics matters hypothesis' instead (Gósy \& Eklund, 2018). What is of interest here is that the relative distribution of PRs seems to be, at least partly, depending on the underlying phonotactics of the language in question, where more permissive phonotactics result in a more even PR distribution in the word. The reason for this change was that Betz, Eklund and Wagner (2017) reported 7-15-78\% for German, and Gósy and Eklund (2018) reported 1819-63 for Hungarian, thus making the overall impression slightly more muddled than the impression given by the previously mentioned studies; for example, German, although more similar to English and Swedish from a morphological and phonotactical point of view, lies closer to Mandarin when it comes to initial PRs, and Hungarian lies closer to Tok Pisin for initial position, than either language does when compared to English or Swedish.

In Hebrew, PRs and FPs were thoroughly studied (Silber-Varod, 2013a; 2013b), however with the current comprehensive speech disfluency framework, the Hebrew PR phenomenon would be comparable to other languages. Hebrew is a Semitic language that belongs to the group of Afro-Asiatic languages, taxonomically different from the previously studied languages. Although classical Hebrew is a fusional language (also called inflected language), with a rich morphology and an extensive system of affixation, Modern Hebrew is considered more analytic compared to rather synthetic Classical Hebrew. The inflected characteristics of Hebrew have implications on the syntactically relevant parts of speech (POS) tag classes. These classes do not 
necessarily correspond to Hebrew orthography. In the morphology and orthography of Hebrew, words are often formed by concatenating smaller parts, which function as free morpho-syntactic units, each of which with its own POS tag. The basic parts are: a potentially polymorphemic stem; a template/pattern morpheme; and possibly an affix. The other morphemes within Hebrew orthographic words are clitics for certain prepositions, conjunctions, definiteness marking, and other POS (Bar-Haim, Sima'An \& Winter, 2008). For the purpose of this study, we defined Hebrew clitics, such as conjunctions, articles, prepositions and so on, as words, following Silber-Varod (2013a).

The phoneme inventory of Hebrew contains five vowels and 26 consonants. There are no short-long phonemic pairs. Hebrew is a 'stressed-timed' language where primary stress can be found on one of the right-most three syllables in the word, with final stress being the most common pattern. The syllable nucleus in Hebrew is always a vowel. Word length is 2.3 syllables on average (SD 0.8) in spontaneous speech (Silber-Varod \& Levy, 2014), and words rarely consist of 6 or more syllables.

The goal of this study is to analyze Hebrew PRs to see to what degree that morphology and syllable structure might influence the distribution of prolonged segments in spontaneous speech of the language. We had three main hypotheses: (i) PRs will occur mostly on the final segments; (ii) PRs will occur mostly on monosyllabic words; (iii) Prolonged vowels will be more frequent than prolonged consonants.

\section{Method and material}

Thirty-six speakers participated in this study. Ages ranged between 21 and 54 years; the median age was 30 years. 21 of the speakers were females. The session began with a structured interview comprising six fixed questions (detailed in SilberVarod et al., 2016). The interviewer was an MA student in clinical psychology. All participants were native speakers of Hebrew living in Israel. There were no indications of hearing, language or speech disorders for any of the participants.

Recordings were made in a sound-attenuated room (the same for all), under identical technical conditions using Sennheiser MKE 2 microphone digitized with an Icicle $48 \mathrm{~V}$ external sound card connected to a computer. The microphone was at a fixed distance from the speaker's mouth, and the recording was carried out with a sampling frequency of $48 \mathrm{kHz}, 16$ bit sample resolution. The duration of the analyzed spontaneous narratives was about 94 minutes (ca. 3 minutes/speaker).

\section{Target segments}

Prolongations were segmented manually by a phonetician (one of the authors), a native Hebrew speaker, using Praat software (Boersma \& Weenink, 2015). Although relying mostly on perception, a minimum threshold of 230 milliseconds was set, based on Silber-Varod (2013a). Vowel boundaries were marked between the onset and offset of the second formants of the vowels. Duration measurements were carried out automatically using Praat's textgrids.

The annotation of prolonged syllables resulted in 347 prolonged segments. The minimal duration was found to be $247 \mathrm{~ms}$ and the maximal $1.964 \mathrm{~s}$. The perceived prolonged segments were then compared to a durational model of Hebrew segments in fluent speech (Modan, 2018: 124, 169-173) and to syllable durations as a function of prosodic environments (Silber-Varod, 2013a: 74-77). The comparison to the fluent durational model ruled out two segments of two different speakers because the ratios between the fluent durational model and the actual prolonged segments were above 50\%. Thus, prolonged segments in the current study are at least twice as long as their fluent counterparts. The comparison to syllable durations as a function of prosodic environments (Silber-Varod, 2013a) ruled out four different vowels, produced by four different speakers, of which two were those ruled out also by Modan's (2018) model, and the other two fluent/prolonged ratios were $36 \%$ and $37 \%$.

On the next step, the remaining 343 prolongations were categorized according to five parameters:

(i) Number of syllables of the word containing the prolonged segment (from 1 to 4);

(ii) Position of the target segment in the word (initial, medial, final, and monosyllabic words that consist of a single vowel);

(iii) Type of segment (vowel vs. consonant);

(iv) Duration of the prolonged segment;

(v) Sex.

\section{Results}

On average, speakers uttered 3.57 PRs per minute (ranging from 0.51 to 7.65 PRs per minute), and in total an average of 9.53 PRs per speaker (variability ranges from 1 to 30). Figure 1 presents these results. The ratio of 343 PRs is $3.5 \%$ of all word tokens.

\section{Number of syllables in words}

Table 1 shows that the number of syllables in the affected words played a role in segment prolongation. There is a strong linear fall-off as a function of number of syllables in the affected words: the fewer the number of syllables, the more likely the word is to exhibit prolongation. 


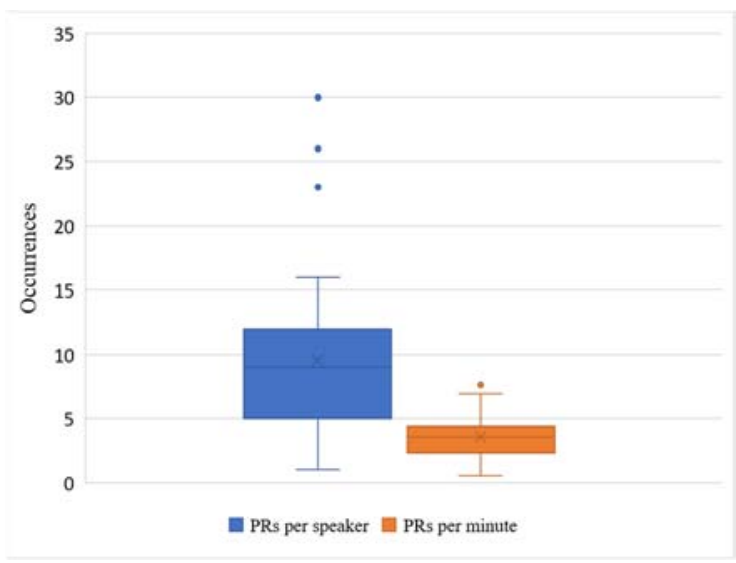

Figure 1: Prolongation distribution per speaker and per minute. Median and mean are indicated.

Table 1. Occurrences and relative frequency of prolongation as a function of number of syllables in the affected word.

\begin{tabular}{|c|c|c|c|c|c|c|c|}
\hline $\begin{array}{c}\text { Number of } \\
\text { syllables in } \\
\text { word }\end{array}$ & $\begin{array}{l}\text { Occurrenc } \\
\text { es of the } \\
\text { words }\end{array}$ & $\begin{array}{c}\text { Relative } \\
\text { frequency } \\
\text { (\%) }\end{array}$ & $\mathbf{C C V}$ & $\mathbf{C V}$ & $\mathbf{C V C}$ & $\mathbf{V}$ & $\mathbf{V C}$ \\
\hline 1 & 254 & 74.05 & 5 & 240 & 1 & 4 & 4 \\
\hline 2 & 51 & 14.87 & 0 & 43 & 6 & 1 & 1 \\
\hline 3 & 37 & 10.79 & 0 & 33 & 2 & 2 & 0 \\
\hline $4^{*}$ & 1 & 0.29 & 0 & 1 & 0 & 0 & 0 \\
\hline Total & $\mathbf{3 4 3}$ & & 5 & 317 & 9 & 7 & 5 \\
\hline
\end{tabular}

* The 4-syllable word is a loan word.

A comparison of the relative frequencies of the syllable per prolonged word in the current study to the ratios that were found in spontaneous Hebrew (Silber-Varod and Levy, 2014), shows higher rates of prolonged monosyllables $(74.05 \%$ in the current study versus $48 \%$ in spontaneous speech), and lower rates of the prolonged words with $>1$ syllables. As to the syllable structure of the prolonged syllables, we found five syllabic structures: CCV, CV, CVC, V, VC. Table 1 presents the distribution of each syllable structure within the number of syllables in the word.

\section{Position}

Regarding the distribution of initial-medial-final segment prolongation, our results show that in Hebrew, $97.95 \%$ of PRs are final, while the other positions are negligible (Table 2). Note that 41 cases of monosyllable $\mathrm{CV}$ words with [h] as the onset were categorized as Final PRs. These cases include the definite article /ha/ 'the', and the two singular thirdperson pronouns /hu/ 'he' and /hi/ 'she'.

Regarding lexical stress, prolongations occur mostly $(74.05 \%)$ on monosyllabic function words that have no assigned stress. Within the 51 disyllabic words, we found $91.48 \%$ PRs on a stressed syllable (for example [aní:] 'I'). Within the 37 tri-syllable words, we found $75.67 \%$ PRs on an unstressed syllable (for example, [anáxnu:] 'we'). In total, PRs occur mostly on unstressed syllables $(84.83 \%)$.
Table 2. PR distribution in words (occurrences)

\begin{tabular}{|l|c|c|c|c|}
\hline Number of syllables in word & Initial & Medial & Final & Single V \\
\hline 1 & 1 & 0 & 249 & 4 \\
\hline 2 & 1 & 1 & 49 & 0 \\
\hline 3 & 0 & 0 & 37 & 0 \\
\hline 4 & 0 & 0 & 1 & 0 \\
\hline Total & $\mathbf{2}$ & $\mathbf{1}$ & $\mathbf{3 3 6}$ & $\mathbf{4}$ \\
\hline
\end{tabular}

\section{Segments}

The prolonged segments are presented in Table 3. All speakers prolonged vowels (Vs), while only 13 speakers prolonged consonants (Cs). Interestingly, the most elongated segment is the vowel [e], which is also the sole realization of filled pauses in Hebrew. In the Cs section, it is not surprising to find the two nasals $[\mathrm{m}]$ and [n], the liquid [1] and two unvoiced fricatives $[\delta]$ and $[\mathrm{x}]$. It is interesting to note that an extremely prolonged [x:] has only recently entered the Hebrew lexicon as an interjection which means LOL (Laugh Out Loud). As is seen, prolongations are distributed in a very restricted manner, as compared to the previously studied languages (see Introduction).

Table 3. Distribution of segments subject to prolongation and relative frequency given as percentages.

\begin{tabular}{|c|c|c|}
\hline $\begin{array}{l}\text { Vowels } \\
(N=324 ; 94.46 \%)\end{array}$ & Occurrences & Relative frequency \\
\hline $\mathrm{e}$ & 166 & $48.40 \%$ \\
\hline $\mathrm{a}$ & 62 & $18.08 \%$ \\
\hline $\mathrm{i}$ & 40 & $11.66 \%$ \\
\hline $\mathrm{u}$ & 35 & $10.20 \%$ \\
\hline $\mathrm{O}$ & 21 & $6.12 \%$ \\
\hline \multicolumn{3}{|l|}{$\begin{array}{l}\text { Consonants } \\
(N=19 ; 5.53 \%)\end{array}$} \\
\hline $\mathrm{m}$ & 12 & $3.50 \%$ \\
\hline 1 & 4 & $1.17 \%$ \\
\hline $\int$ & 1 & $0.29 \%$ \\
\hline $\mathrm{n}$ & 1 & $0.29 \%$ \\
\hline $\mathrm{x}$ & 1 & $0.29 \%$ \\
\hline
\end{tabular}

\section{Duration of the prolonged segments}

In Figure 2, we show the results of our durational analysis, broken down for Vs and Cs. As in the previous calculation, here also we first averaged prolonged Vs and prolonged Cs per speaker, and then across speakers. The average duration of the prolonged Vs is 0.57 seconds and the average duration for prolonged $\mathrm{Cs}$ is 0.51 seconds. A two-tailed $t$-test showed no significant differences between the durations of Vs and Cs $(p=0.72)$.

\section{Sex}

In total, men produced $52 \%$ of the prolongations (179 versus 163 that women did), with a standard deviation of 6.69 for men and 5.89 for women. On average, men produced 11.93 PRs per interview, while women only 7.45 PRs. A one-tailed $t$-test showed that this difference was statistically 
significant $(p=0.03)$. Regarding duration, on average, PR segments produced by men are 0.599 seconds and PR segments produced by women are 0.540 . In an unpaired $t$-test, this difference was not found to be statistically significant $(p=0.19)$.

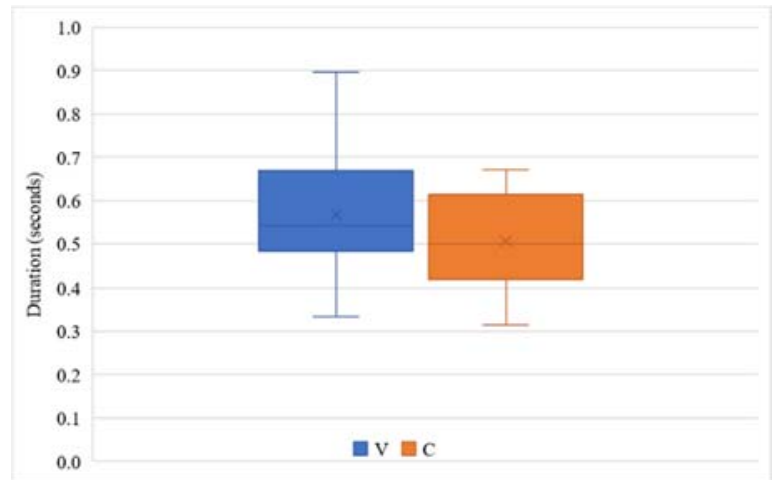

Figure 2. Durations of PRs broken down for vowels and consonants.

\section{Discussion and conclusions}

In this paper, an analysis of 343 PRs in spontaneous Hebrew resulted in several generalizations: Regarding Distribution, Hebrew prolongations occur $97.95 \%$ on the final segment and scarcely on initial or medial segments. Interestingly, this makes $\mathrm{He}-$ brew more similar to Tok Pisin, Japanese and Mandarin Chinese than to English, German or Swedish, with a roughly $1-1-98 \%$ distribution. As for Segments, vowels are, on the whole and perhaps not surprisingly, more frequently prolonged than consonants in our data, unlike American English and Swedish. Regarding stress, PRs occur mostly on unstressed syllables. As for Duration, we did not find any effect of segment type (vowel or consonant) on the durations of PRs. As for Sex, there is a significant tendency for male speakers to produce more PRs than female speakers do. Assuming that PRs are a strategy to keep the floor, these findings support the hypothesis that men are less prone to yielding the floor in dialog (see Eklund \& Wirén, 2010: 23).

\section{Acknowledgements}

We are grateful for Hamutal Kreiner from Ruppin Academic College for funding the transcriptions.

\section{References}

Bar-Haim, R., K. Sima'An \& Y. Winter. 2008. Part-ofspeech tagging of Modern Hebrew text. Natural Language Engineering 14(2): 223-251. https://doi.org/10.1017/S135132490700455X

Betz, S., R. Eklund \& P. Wagner. 2017. Prolongation in German. In: R. Eklund \& R. Rose (eds.), Proceedings of DiSS 2017, the 8th Workshop on Disfluency in Spontaneous Speech, 18-19 August 2017, KTH Royal Institute of Technology, Stockholm Sweden, TMHQPSR Volume 58(1): 13-16.
Boersma, P. \& D. Weenink. 2015. Praat: Doing phonetics by computer (ver. 6.0.41) http://www.praat.org/ (Accessed 25 August 2018).

Den, Y. 2003. Some strategies in prolonging speech segments in spontaneous Japanese. In: R. Eklund (ed.), Proceedings of DiSS'03, Disfluency in Spontaneous Speech, 5-8 September 2003, Göteborg, Sweden. Gothenburg Papers in Theoretical Linguistics 90, 87-90.

Eklund, R. 2001. Prolongations: A dark horse in the disfluency stable. In: Proceedings of DISS 2001, Disfluency in Spontaneous Speech. Edinburgh, Scotland, 5-8.

Eklund, R. 2004. Disfluency in Swedish human-human and human-machine travel booking dialogues. Ph.D. dissertation, Linköping University, Sweden.

Eklund, R. \& E. Shriberg. 1998. Crosslinguistic Disfluency Modelling: A Comparative Analysis of Swedish and American English Human-Human and Human-Machine Dialogues. Proceedings of ICSLP 98, 30 November-5 December 1998, Sydney, Australia, vol. 6, 2631-2634.

Eklund, R. \& M. Wirén. 2010. Effects of open and directed prompts on filled pauses and utterance production. In: Proceedings of Fonetik 2010, Lund, Sweden, 23-28.

Gósy, M. \& R. Eklund. 2017. Segment prolongation in Hungarian. In: R. Eklund \& R. Rose (eds.), Proceedings of DiSS 2017, the 8th Workshop on Disfluency in Spontaneous Speech, KTH Royal Institute of Technology, Stockholm Sweden, TMHQPSR Volume 58(1): 29-32.

Gósy, M. \& R. Eklund. 2018. Language-specific patterns of segment prolongation in Hungarian. The Phonetician, 115, 36-52.

Lee, T.-L., Y.-F. He, Y.-J. Huang, S.-C. Tseng \& R. Eklund. 2004. Prolongation in spontaneous Mandarin. In: Proceedings of Interspeech 2004, Jeju Island, Korea, vol. III: 2181-2184.

Siptár, P. \& M. Törkenczy. 2000. The phonology of Hungarian. Oxford: Oxford University Press.

Modan, D. 2018. Models for the prediction of durations in Hebrew Speech. Ph.D. dissertation, The Hebrew University of Jerusalem.

Silber-Varod, V. 2013a. The SpeeCHain Perspective: Form and Function of Prosodic Boundary Tones in Spontaneous Spoken Hebrew. LAMBERT Academic Publishing. https://doi.org/10.1075/la.215.10sil

Silber-Varod, V. 2013b. Structural analysis of prosodic pattern: The case of excessive prolongations in Israeli Hebrew. Revista Leitura, Special Issue on Speech Prosody 52: 271-291. https://doi.org/10.28998/2317-9945.2013v2n52p271-291

Silber-Varod, V., H. Kreiner, R. Lovett, Y. Levi-Belz \& N. Amir. 2016. Do social anxiety individuals hesitate more? The prosodic profile of hesitation disfluencies in Social Anxiety Disorder individuals. Proceedings of Speech Prosody 2016. 31 May-3 June 2016, Boston, USA, 1211-1215. https://doi.org/10.21437/SpeechProsody.2016-249

Silber-Varod, V.\& T. Levy. 2014. Intonation Unit Size in Spontaneous Hebrew: Gender and Channel Differences. Proceedings of Speech Prosody 7, 20-23 May 2014, Dublin, Ireland, 658-662. 